\title{
Conceptual design and virtual prototyping of a wearable upper limb exoskeleton for assisted operations
}

\author{
Pietro Bilancia ${ }^{1}$ (D) Giovanni Berselli ${ }^{2}$ (D) \\ Received: 30 December 2020 / Accepted: 20 September 2021 / Published online: 22 October 2021 \\ (c) The Author(s) 2021
}

\begin{abstract}
This paper introduces a novel upper limb robotic exoskeleton designed to assist industrial operators in a wide range of manual repetitive tasks, such as tool handling and lifting/moving of heavy items. Due to its reduced size and high maneuverability, the proposed portable device may also be employed for rehabilitation purposes (e.g. as an aid for people with permanent neuromuscular diseases or post-stroke patients). Its primary function is to compensate the gravity loads acting on the human shoulder by means of a hybrid system consisting of four electric motors and three passive springs. The paper focuses on the exoskeleton mechanical design and virtual prototyping. After a preliminary review of the existent architectures and procedures aimed at defining the exoskeleton functional requirements, a detailed behavioral analysis is conducted using analytical and numerical approaches. The developed interactive model allows to simulate both kinematics and statics of the exoskeleton for every possible movement within the design workspace. To validate the model, the results have been compared with the ones achieved with a commercial multibody software for three different operator's movements.
\end{abstract}

Keywords Upper limb exoskeleton · Computer aided design · Virtual prototyping $\cdot$ Gravity balancing $\cdot$ Robotic arm

\section{Introduction}

In recent years, the interest for assistive devices has been increasing due to their proven effectiveness in many cardinal areas, such as medical treatments [1-4] and industrial manufacturing processes [5-8]. These advanced mechanical/mechatronic systems are gradually stepping from the research labs to the real life applications, mainly for the rehabilitation of injured or disabled patients $[9,10]$, but also for enhancing the operators performance in many work environments [11]. Under the trend of Industry 4.0 [1214], the level of automation is constantly increasing in the production lines, though many manual repetitive tasks (e.g. tool handling, time-consuming overhead operations,

Giovanni Berselli

giovanni.berselli@unige.it

Pietro Bilancia

pietro.bilancia@unimore.it

1 DISMI-Department of Sciences and Methods for Engineering, University of Modena and Reggio Emilia, Via Giovanni Amendola, 2, 42122 Reggio Emilia, Italy

2 DIME-Department of Mechanics, Energetics, Management and Transports, University of Genova, Via all'Opera Pia, 15, 16145 Genova, Italy machine loading/unloading, lifting/carrying of heavy components, operations under asymmetric un-ergonomic body postures, etc.), which cannot be automated, are still existent at large scale $[15,16]$. They become arduous, exhausting or even dangerous after a certain amount of time. In fact, workers exposed to these activities are more likely to manifest work-related musculoskeletal disorders [17,18].

Within this scenario, the development of portable, lightweight and ergonomic assistive devices seems to be an effective strategy to reduce the risk of injuries in manual manufacturing and also to avoid loss of productivity [5]. Their primary function is to assist people during the execution of specific movements by providing supplementary strength, making it possible, for instance, to deal with greater loads, to compensate for a lack of muscularity and to prevent from excessive fatigue. Wearable assistive devices, usually referred to as exoskeletons, are commonly designed to operate along their human counterparts, namely the lower/upper limbs or the neck area, though in the last case, if no particular functionalities are provided, the device could be better identified as a brace [24]. Ready examples of commercially available exoskeletons are the Mate, [19], the AirFrame [20] and the ShoulderX [21] for the upper limbs, the $\operatorname{LegX}$ [22] and the Hercule [25] for the lower limbs, and the Laevo V2 


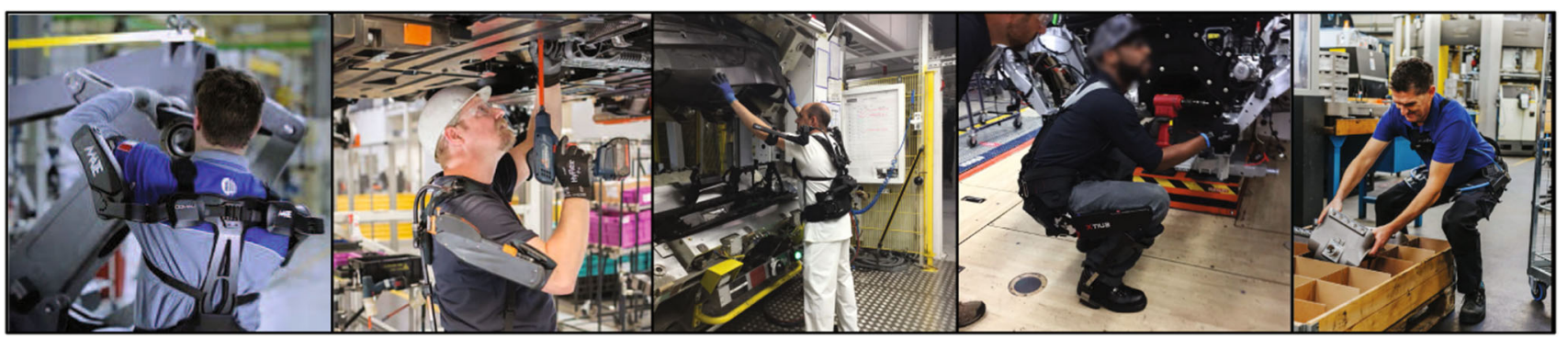

Fig. 1 Examples of assistive devices for manual tasks in the manufacturing industry. From the left: the Mate [19], the AirFrame [20], the ShoulderX [21], the LegX [22] and the Laevo V2 [23]

[23] for the lumbar region. Despite these promising solutions, shown in Fig. 1, have not yet been fully integrated in the industrial sector, many pilot studies highlighted their positive effects on the operators, which are well documented in $[5,8,11,26,27]$. Similarly, also soft exoskeletons (e.g. arm/leg exosuits [28,29]) represent an important research topic, with several prototypes currently transitioning from lab to market. In this case, the system shall comprise deformable sensory-motor technologies, such as soft/compliant [30,31] and sensors [32]).

Generally, the design of an efficient exoskeleton is a challenging task since it requires a deep knowledge of the biomechanics of human movements, which are often not simple and may involve many joints and parts [33]. From a kinematic standpoint, an exoskeleton must be quasiequivalent to the related human limb, otherwise undesired motion and force contributes would be introduced during the functioning and then transmitted to the wearer, causing discomfort or even diseases [34]. These design issues are emphasized when considering the human upper limbs, because they are smaller than the lower limbs and they have mobility in a wide range of space, as clearly described in [35]. In particular, it shall be remarked that the shoulder is one of the most anatomically intricate region of the human body, as it combines four joints (i.e. the Glenohumeral joint, the Acromioclavicular joint, the Sternoclavicular joint and the Scapulothoracic joint, as shown in Fig. 2) and a large number of bones, muscles, ligaments and tendons [36]. Also, because of the intrinsic flexibility of the human musculoskeletal system, which varies between individuals based on their age, sex and state of health, a general parametric kinematic model cannot be easily formulated for the shoulder complex. Starting from [37], where the shoulder is simplified to a ball and socket joint, plenty of kinematic configurations have been proposed and analyzed. Most of these utilize serial chain manipulators and adopt a 3 Degrees of Freedom (DoFs) design for the Glenohumeral joint, where the axes of the 3 Revolute (R) joints must intersect the human shoulder center of rotation [3,4,38-42]. Other configurations employ $4 R[43,44]$ or $5 R$ [34] redundant schematics with the aim of overcoming pos-

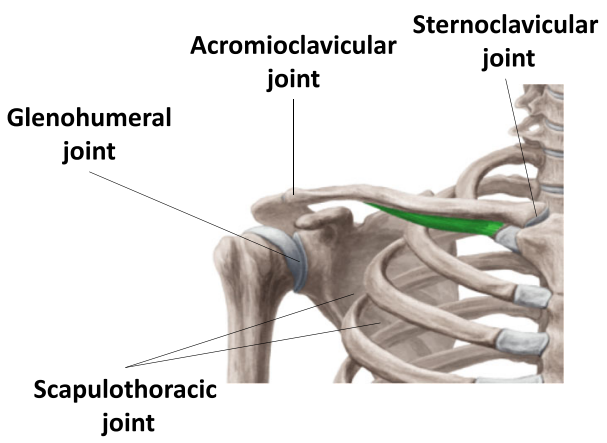

Fig. 2 Biomechanics of the human shoulder

sible singular configurations. Alternative mechanical layouts can be found in [45], in [46], and in [47], where a double parallelogram linkage, a hyper-redundant chain and a scissors linkage are respectively proposed. Given that the exoskeleton is attached to the human arm and must resemble its motion, a standard design rule is to add extra DoFs in order to better follow the human shoulder's broad variety of activities and avoid misalignment between human joints and exoskeleton joints. For a comprehensive review of the existent hardware in the field of upper limb exoskeletons, the interested reader may refer to [48-50].

Besides the mechanism topology, another crucial point for the exoskeleton design is the selection of the balancing principle. According to the literature, the exoskeletons can be classified into active or passive types based on the presence or not of externally-powered actuators in the assisted mechanism [48]. The former provide reactive and precise assistance via the combined use of a sensory apparatus, a set of motors and a controller, whereas the latter rely on the presence of passive spring-like components to counterbalance the loads due to gravity. At the early design stage, the proper selection between them should be guided by the following points:

- Biomechanics of the human limb-A single DoF kinematic chain, such as the elbow joint, can adopt either active [52] or passive [53] balancing without any restriction. Obviously, in case of active balancing, the motor 


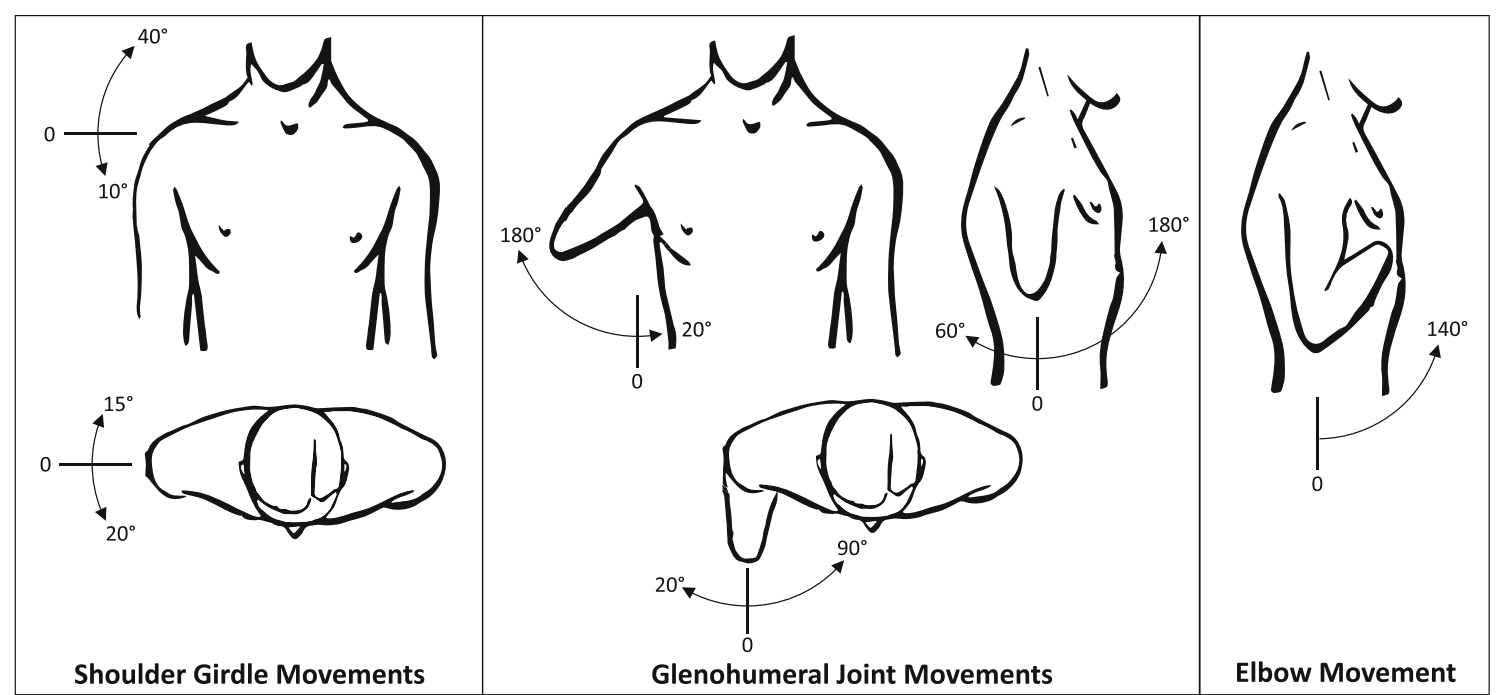

Fig. 3 Typical movements of the human upper limbs [51]

placement is to be carefully investigated in order to increase the overall compactness and ensure a sufficient level of mobility. Instead, when considering more complex scenarios, namely human limbs whose motions are unquestionably to be obtained by means of multiDoFs chains (e.g. the shoulder joint), the designer may encounter issues in terms of maneuverability if an active balancing system is chosen because of the presence of many additional components installed therewith. A partial solution for this problem is the use of a remote actuation and cable-driven transmissions [50,54,55]. In any case, the overall assembly would be surely simplified if a passive system is implemented, as in [4].

- Balancing action-This aspect is strictly related to the field of application. As previously mentioned, the exoskeletons have potentials to be utilized in many situations, among which the assistance of workers that face repetitive tasks, but also the rehabilitation of stroke patients or the treatment of people with neuromuscular disorders. Therefore, on the basis of the specific assistance being provided, the exoskeleton balancing is expected to be more in static or dynamic conditions. Slow movements fall into the static balancing and leave the designer free to select either active or passive systems, whereas rapid movements are most likely to be assisted via a motorized solution. For industrial-oriented exoskeletons, an interesting study showing the priority joints for active assistance to prevent back and shoulder injuries can be found in [56].

- Balancing accuracy-While passive exoskeleton are usually constrained to a specific set of target tasks, the active balancing can be achieved for a broad range of movements by means of the sensory feedback and a rapid control action [57].
- Overall weight - As it may be expected, large and heavy linkages may need the use of motors to receive an adequate assistance. This is the case of heavy rehabilitation prototypes, as the ones visible in $[3,37]$. Conversely, the passive balancing is promoted when light-weight and compact designs are required.

By combining these features, one could also synthesize hybrid exoskeletons, namely systems comprising both motorized joints and passive elastic elements [58]. These solutions allow to select smaller actuators, reducing the risk of collision between the components and facilitating the interaction with the wearer.

In this context, this paper aims to propose a novel portable, hybrid, interactive, upper limb exoskeleton to be used in both industrial and healthcare environments to reduce the human musculoskeletal loads. The idea is to combine the advances, in terms of mechanism efficiency, visible in recent academic research prototypes, with the benefits of the existent commercially available exoskeletons (such as the ones in Fig. 1), which can be outlined into simplified assembly, reduced weight and limited production costs. Therefore, the proposed exoskeleton makes use of a limited number of parts, which leads to a relatively simple structure. It follows the natural motions of the human shoulder (including the contributes from the shoulder girdle) and the elbow joints, i.e. the ones visible in Fig. 3. The balancing is achieved via the concurrent action of four electric DC motors and three linear springs placed between the rigid links. The paper focuses on the mechanical design of the device and describes its main characteristics. The exoskeleton kinematics and statics are considered in details. In particular, an analytical model is developed with the aim of determining the overall range 


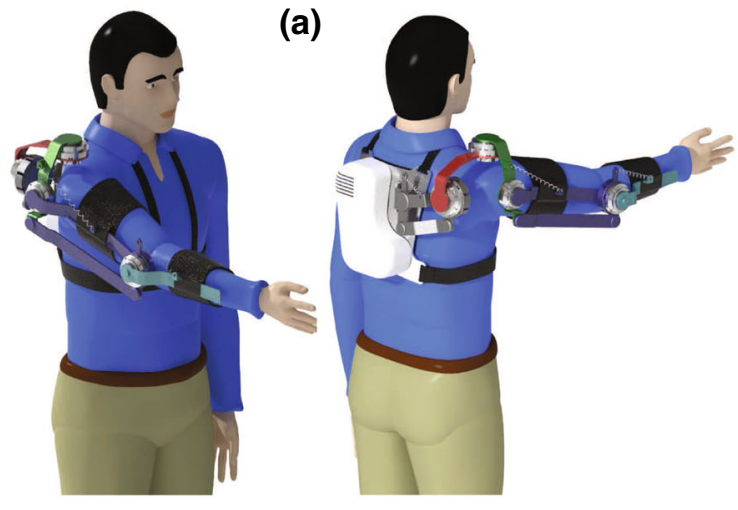

(c)

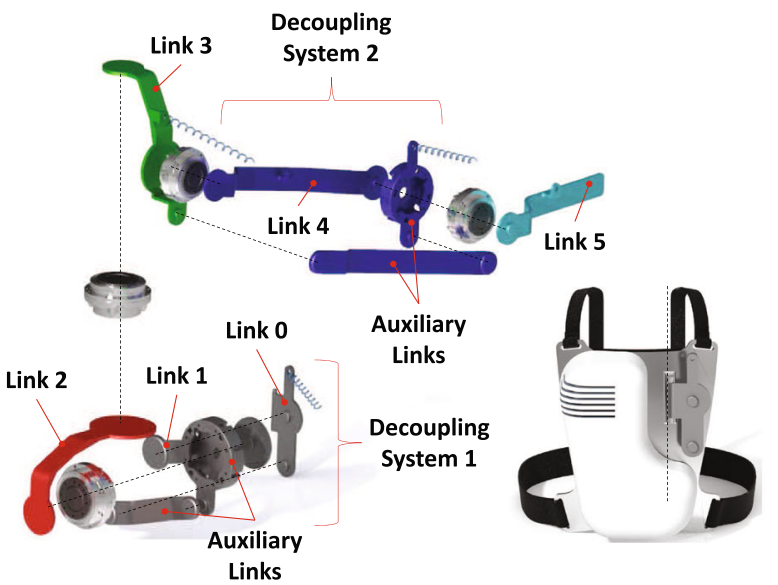

(b)

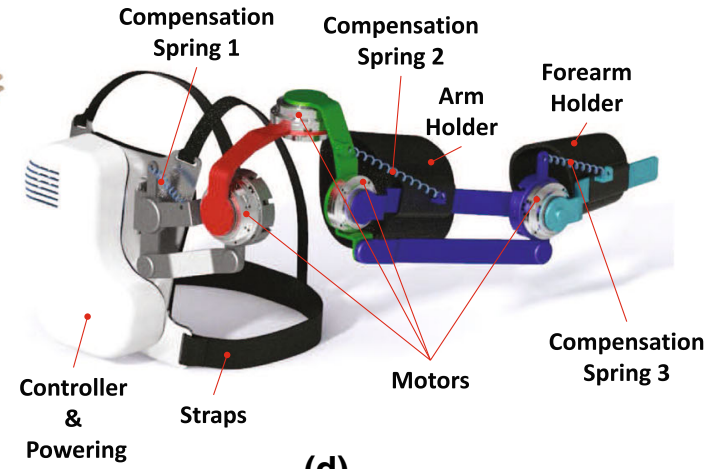

(d)

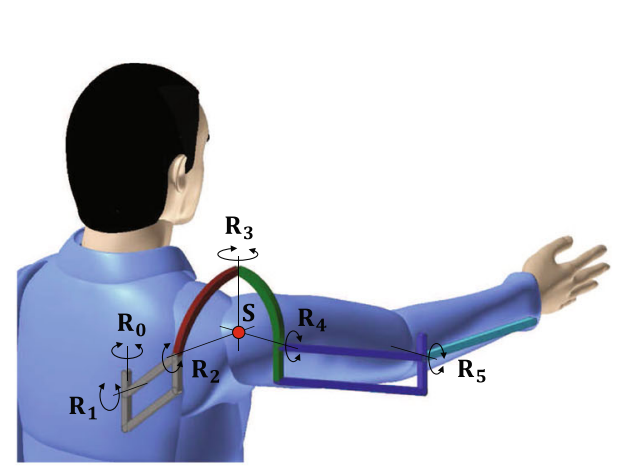

Fig. 4 Proof-of-concept design of the exoskeleton. a Example of the right-arm version on a manikin. b Detailed view of the main components. $\mathbf{c}$ Exploded CAD view. d Kinematic model and its DoFs

of motion as well as the correct size of the motors and of the springs. Also, this parametric and efficient interactive model will surely be of interest for the controller design of the exoskeleton. As a last step, the model is validated through a commercial Multibody Dynamics (MBD) software under different working conditions.

The rest of the paper is structured as follows: Sect. 2 describes the main features of the proposed exoskeleton design, Sect. 3 recalls the background theory of planar balancers, Sect. 4 reports the developed analytical model and its numerical validation, whereas Sect. 5 provides the concluding remarks.

\section{Design concept}

For the design of the exoskeleton system, a number of features have been considered. First, in view of its use as a multi-tasking portable device, the compactness as well as the total weight and the user safety become primary concerns [59]. Second, the device must achieve high dexterity so as not to cause impediments to the user. This applies especially to the industrial devices (see Fig. 2), because rehabilitative devices usually need a smaller mobility and their design is focused on the motion accuracy. The embodiment design of the developed exoskeleton is described hereinafter. In particular, Fig. 4a shows the right-arm configuration of the portable device mounted on a manikin, whereas Fig. 4b and $\mathrm{c}$ provide a detailed view of its main components. As visible in these figures, the overall structure is compact and is connected to the human arm by means of two cuffs (i.e. Arm Holder and Forearm Holder). The kinematic model is depicted in Fig. 4d, where a simplified version of the overall exoskeleton is schematized. Differently from industrial solutions employing simplified kinematic chains (see, e.g., $[19,20])$, the proposed mechanism has 6 DoFs for supporting the motion of the human shoulder and the elbow joints. With reference to Fig. $3,2 \mathrm{R}$ joints $\left(R_{0}\right.$ and $R_{1}$ in Fig. 4d) are used to model the shoulder girdle movements, $3 \mathrm{R}$ joints $\left(R_{2}, R_{3}\right.$ and $R_{4}$ in Fig. $\left.4 \mathrm{~d}\right)$ provide the glenohumeral movements, and the last $\mathrm{R}$ joint $\left(R_{5}\right.$ in Fig. $\left.4 \mathrm{~d}\right)$ is aligned with the elbow joint. Two parallelogram decoupling systems (Fig. 4c) have been included in the kinematic chain, making it possible to simplify the passive balancing [60], 


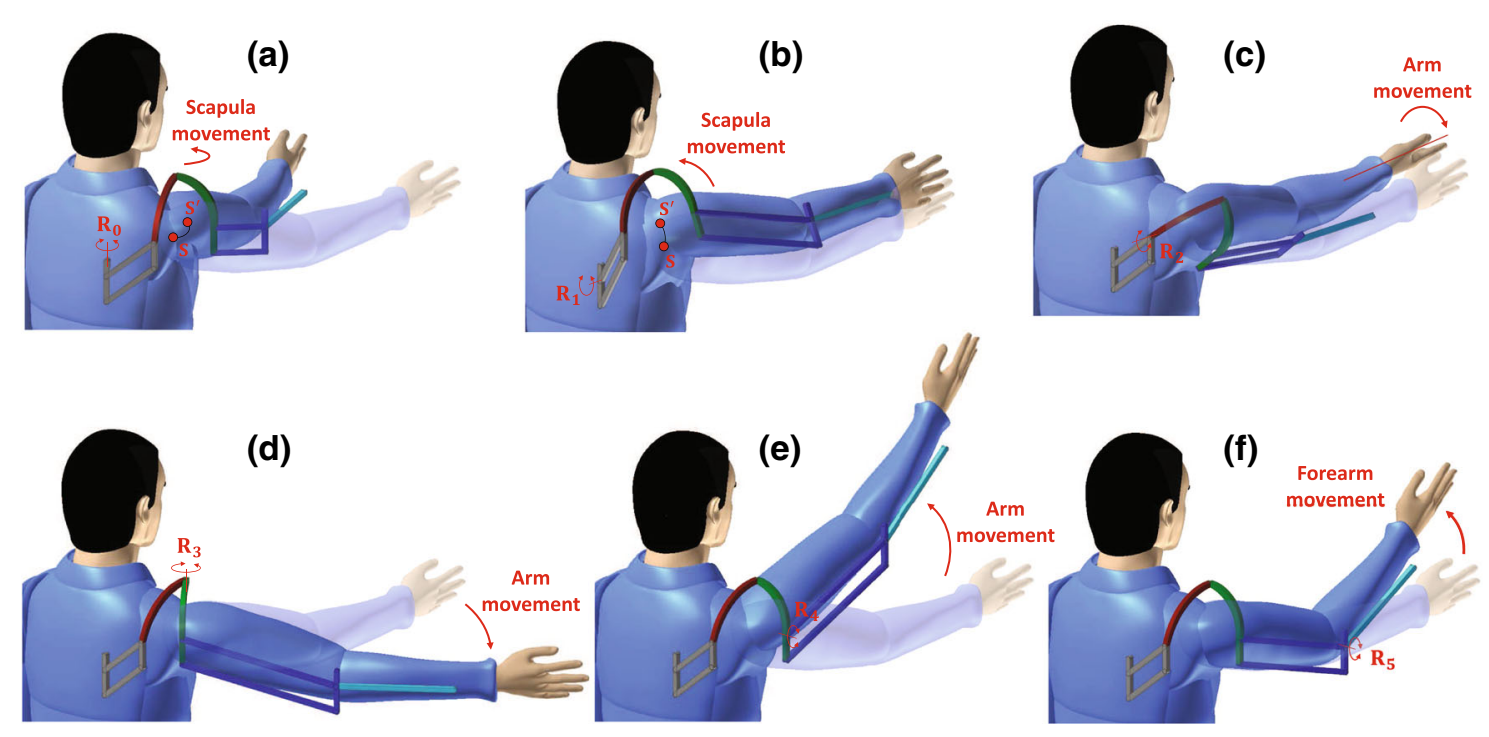

Fig. 5 Exoskeleton primary motions. Single effect of $R_{0}(\mathbf{a}), R_{1}(\mathbf{b}), R_{2}(\mathbf{c}), R_{3}(\mathbf{d}), R_{4}(\mathbf{e})$ and $R_{5}(\mathbf{f})$

as it will be explained in the next section. Similar solutions can be found in $[3,42]$. The basic motions of the exoskeleton, obtained by rotating a single $\mathrm{R}$ joint (from $R_{0}$ to $R_{5}$ ) while keeping the others fixed, are illustrated in Fig. 5. As visible in Figs. 5a and b, the exoskeleton can capture the glenohumeral joint deviations (displacement from $S$ to $S^{\prime}$ ) in case of scapular abduction/adduction and shoulder girdle elevation/depression, which is a desirable quality, as clearly remarked in [61]. Naturally, to ensure a safety interaction with the operator, mechanical stoppers are implemented to limit the operative range of each $\mathrm{R}$ joint.

As for he gravity balancing, this is obtained by combining:

- Four electric motors, placed in $R_{2}, R_{3}, R_{4}$ and $R_{5}$. Among the available products on the market, the FLA-series of brushless DC motors (by Harmonic Drive SE), equipped with harmonic drive reducers, are here chosen mainly for their small size and high delivered torque. Clearly, the final selection from the manufacturer's catalogs depends on the required torque, which in turn depends on the subject (and task) being assisted.

- Three Non-Zero Free Length (NZFL) linear springs, arranged between Link 0 and Link 1, Link 3 and Link 4, Auxiliary Link and Link 5. Ideally speaking, the Zero Free Length (ZFL) springs are preferable due to their simplified modeling and synthesis. However, they are not readily available and their practical realization requires the use of a wire-and-pulley mechanism [62], that is why many researches utilize NZFL springs [42].

Starting from the background theory, the above discussed points will be analyzed throughout the next sections.

\section{Background on passive balancers}

This section briefly summarizes the main results of previous studies in the field of passive balancers and extrapolates simple formulas that can help the designers in the synthesis process.

\subsection{DoF balancer}

The 1 DoF balancer [63] has been utilized as a basic module in many researches [64-66]. With reference to Fig. 6a, assuming that the link has null density, the torque produced by the mass placed at distance $a$ from the ideal R joint, under the effect of gravity, is equal to:

$M=m g a \sin \theta$

where $\theta$ represents the angular position of the link. By attaching an ideal ZFL linear spring between the ground and the link through two R joints, respectively at distance $c$ and $b$ from the principal $\mathrm{R}$ joint, as in Fig. $6 \mathrm{~b}$, the new moment equilibrium is as follows:

$m g a \sin \theta-k d b \sin \alpha=0$

From the triangle of sides $b c d$, the following trigonometric correlation can be defined:

$c \sin \theta=d \sin \alpha \rightarrow d=\frac{c \sin \theta}{\sin \alpha}$ 
(a)

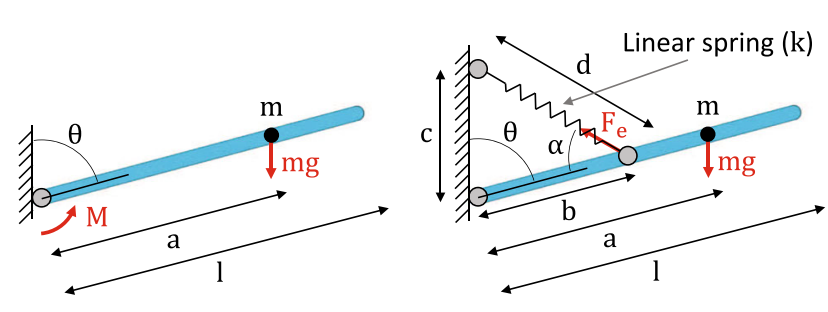

(c)

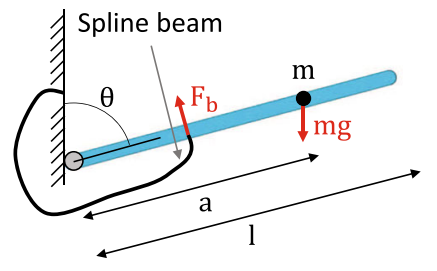

Fig. 6 Planar 1 DoF balancer. a Gravity load acting on the system. b Balancing with a linear spring. c Balancing with a compliant spline beam

By substituting Eqs. 3 in 2, the angles are elided and the expression of the spring stiffness becomes:

$k=\frac{m g a}{b c}$

or, if $a=b$ :

$k=\frac{m g}{c}$

Since the ZFL condition has practical limits, in the following two more solutions are discussed. The first considers spring with NZFL [67]. In this case, by assuming $d_{0}$ as the initial spring length, Eq. 2 can be rewritten as:

$m g a \sin \theta-k\left(d-d_{0}\right) b \sin \alpha=0$

Then, by introducing Eq. 3, a new expression for $k$ can be derived, i.e.:

$k=\frac{m g a}{b c\left(1-\frac{d_{0}}{d}\right)}$

where $d=\sqrt{b^{2}+c^{2}-2 b c \cos \theta}$ from the cosine theorem. To remove the dependence on $\theta$, one may consider $d=\sqrt{b^{2}+c^{2}}$, though this is valid if $\left|\frac{2 b c}{b^{2}+c^{2}}\right| \leq 1$. Under this condition, the spring stiffness becomes:

$k=\frac{m g a}{b c\left(1-\frac{d_{0}}{\sqrt{b^{2}+c^{2}}}\right)}$

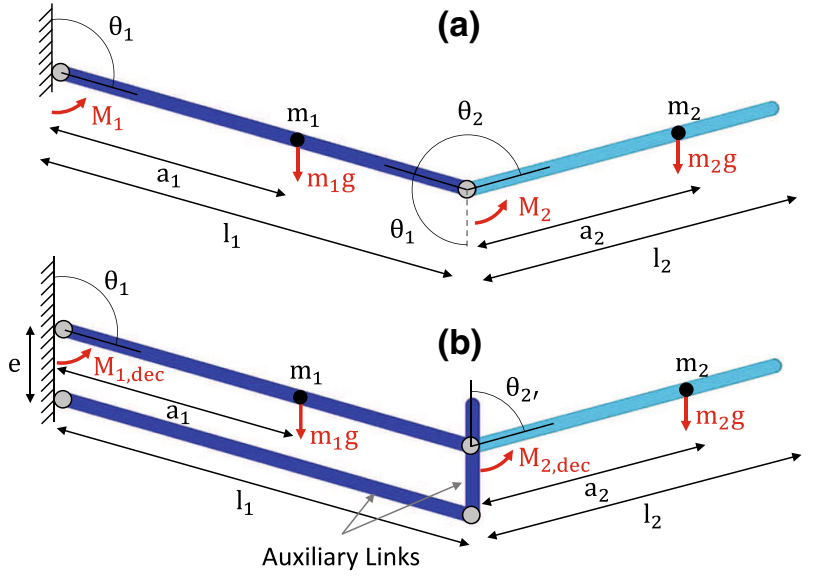

(c)

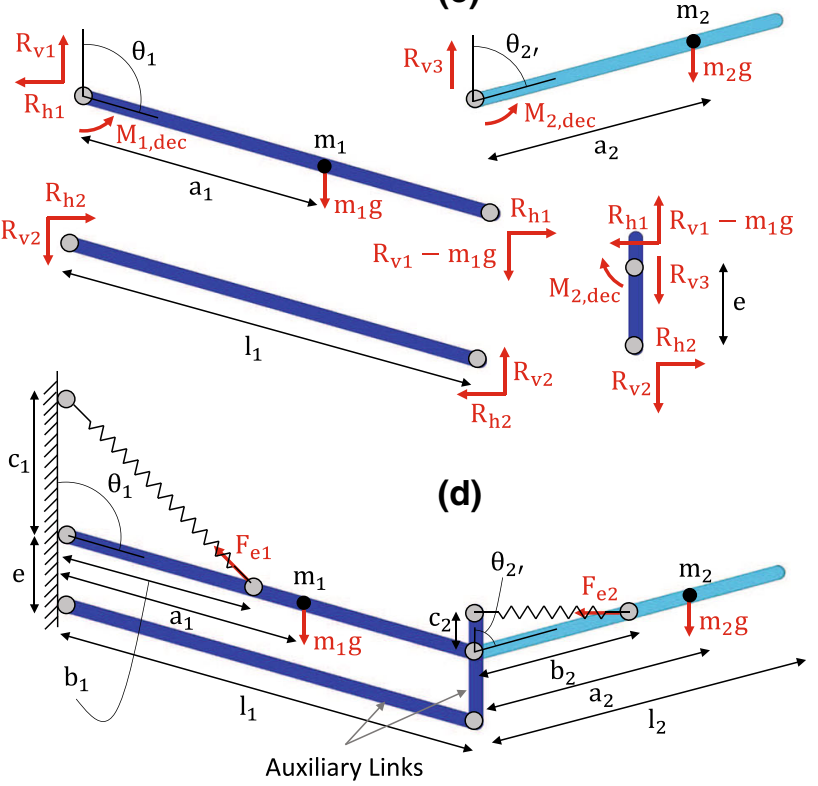

Fig. 7 Planar 2 DoF balancer. a Classic 2 links mechanism. b Decoupling mechanism. $\mathbf{c}$ Static analysis of the decoupling mechanism with external gravity forces. $\mathbf{d}$ Balancing with 2 linear springs

Note that for $d_{0}=0$, Eq. 8 coincides with Eq. 4. Quoting $[53,60]$, another possible way to reach the gravity balancing is the use of properly designed compliant mechanisms [68]. For instance, Fig. 6c shows a possible configuration of compliant balancer, namely a flexible beam whose shape is to be optimized in order to match a specific, pre-defined, force-deflection behavior $\left(F_{b}=F_{b}(\theta)\right)$ in the angular range of interest [69-71].

\subsection{DoFs balancer with decoupled architecture}

For a 2 DoFs linkage, as the one depicted in Fig. 7a, an additional spring element (or, alternatively, a compliant mechanism) is needed. The torques due to gravity are given by: 


$$
\begin{aligned}
M_{1}= & m_{1} g a_{1} \sin \theta_{1}+m_{2} g\left(l_{1} \sin \theta_{1}\right. \\
& \left.-a_{2} \sin \left(\theta_{1}+\theta_{2}\right)\right)= \\
= & m_{1} g a_{1} \sin \theta_{1}+m_{2} g l_{1} \sin \theta_{1}+M_{2} \\
M_{2}= & -m_{2} g a_{2} \sin \left(\theta_{1}+\theta_{2}\right)
\end{aligned}
$$

As it can be noted from Eqs. 9 and 10, both $M_{1}$ and $M_{2}$ are functions of $\theta_{1}$ and $\theta_{2}$, making it problematic to compensate the first/second $\mathrm{R}$ joint (see Fig. 7a) without taking into consideration the position of the other. To overcome this issue, two additional links (Auxiliary Links in Fig. 7b) can be added to form a parallelogram decoupling mechanism [60]. The direct advantages of such decoupling architecture can be deduced from the static analysis of Fig. 7c. In particular, Eq. 9 can be reformulated as:

$M_{2, d e c}=m_{2} g a_{2} \sin \theta_{2^{\prime}}$

The rotational equilibrium of the auxiliary links gives:

$R_{v 2} l_{1} \sin \theta_{1}=R_{h 2} l_{1} \cos \theta_{1} \rightarrow R_{v 2}=R_{h 2} \frac{\cos \theta_{1}}{\sin \theta_{1}}$

and

$M_{2, d e c}=e R_{h 2} \rightarrow R_{h 2}=\frac{M_{2, d e c}}{e}$

By knowing that $R_{v 3}=m_{2} g$ and by considering Eqs. 12 and 13 , the vertical equilibrium of the second auxiliary link becomes:

$R_{v 1}-m_{1} g=m_{2} g+\frac{M_{2, d e c}}{e} \frac{\cos \theta_{1}}{\sin \theta_{1}}$

Now, being $R_{h 1}=R_{h 2}$, the expression of $M_{1, \text { dec }}$ can be derived as follows:

$$
\begin{aligned}
M_{1, \text { dec }} & =m_{1} g a_{1} \sin \theta_{1}+\left(m_{2} g+\frac{M_{2, d e c}}{e} \frac{\cos \theta_{1}}{\sin \theta_{1}}\right) l_{1} \sin \theta_{1} \\
& -\frac{M_{2, d e c}}{e} l_{1} \cos \theta_{1} \\
& =m_{1} g a_{1} \sin \theta_{1}+m_{2} g l_{1} \sin \theta_{1}
\end{aligned}
$$

The effectiveness of the decoupling mechanism can be verified with a rapid comparison between Eqs. 9-10 and Eqs. 11-15. In case of NZFL springs (Fig. 7d), the stiffness constants are determined through the following formulas (obtained by reiterating the steps from Eqs. 2 to 8 with the new expressions of $M_{1, \text { dec }}$ and $M_{2, \text { dec }}$ ):

$$
\begin{aligned}
& k_{1}= \frac{m_{1} g a_{1}+m_{2} g l_{1}}{b_{1} c_{1}\left(1-\frac{d_{01}}{\sqrt{b_{1}^{2}+c_{1}^{2}}}\right)} \\
& k_{2}=\frac{m_{2} g a_{2}}{b_{2} c_{2}\left(1-\frac{d_{02}}{\sqrt{b_{2}^{2}+c_{2}^{2}}}\right)}
\end{aligned}
$$

where $d_{01}$ and $d_{02}$ are the initial free lengths, to be considered null in case of ZFL springs.

\section{Virtual prototype of the exoskeleton}

In the following, an interactive model is developed for studying the exoskeleton kinematics and statics in the design workspace. The mechanism schematic is shown in Fig. 8 and its main dimension parameters are summarized in Table 1. The length of each link (especially the ones attached to the operator's arm and the forearm, i.e. $l_{4}$ and $l_{5}$ ) have been decided in accordance with the work presented in [72], considering a male whose body mass is $80 \mathrm{~kg}$ and height is $1.8 \mathrm{~m}$. From the same study, the operator's arm and forearm masses, and also their center of mass positions, have been determined. Obviously, these parameters can be varied and the model updated in case of different anatomical characteristics.

\subsection{Kinematic model}

The kinematic model of the 6 DoFs exoskeleton can be constructed based on the D-H method [73]. In particular, having defined a coordinate system for each link, $C S_{i}, i \in[0,6]$ (see Fig. 8), a set of six $4 \times 4$ homogeneous transformation matrices, $A_{i}\left(\theta_{i}\right), i \in[0,5]$, can be easily formulated. Following the kinematic chain's rule, being ${ }^{6} \mathbf{q}=\left[\begin{array}{llll}0 & 0 & 0 & 1\end{array}\right]^{T}$ the position vector of the end-effector with respect of the local coordinate system $C S_{6}$, the position vector with respect of the Global Coordinate System ( $G C S$, aligned with $C S_{0}$ only in the initial configuration), $\mathbf{Q}=\left[\begin{array}{llll}X & Y & Z & 1\end{array}\right]^{T}$, is obtained as:

$\mathbf{Q}=\mathbf{A}^{6} \mathbf{q}$

being $\mathbf{A}$ the overall transformation matrix, calculated as:

$\mathbf{A}=\mathbf{\mathbf { A } _ { 0 }} \mathbf{A}_{\mathbf{1}} \mathbf{A}_{2} \mathbf{A}_{3} \mathbf{A}_{4} \mathbf{A}_{5}$ 
Fig. 8 Exoskeleton mechanism schematic with global and local coordinate systems

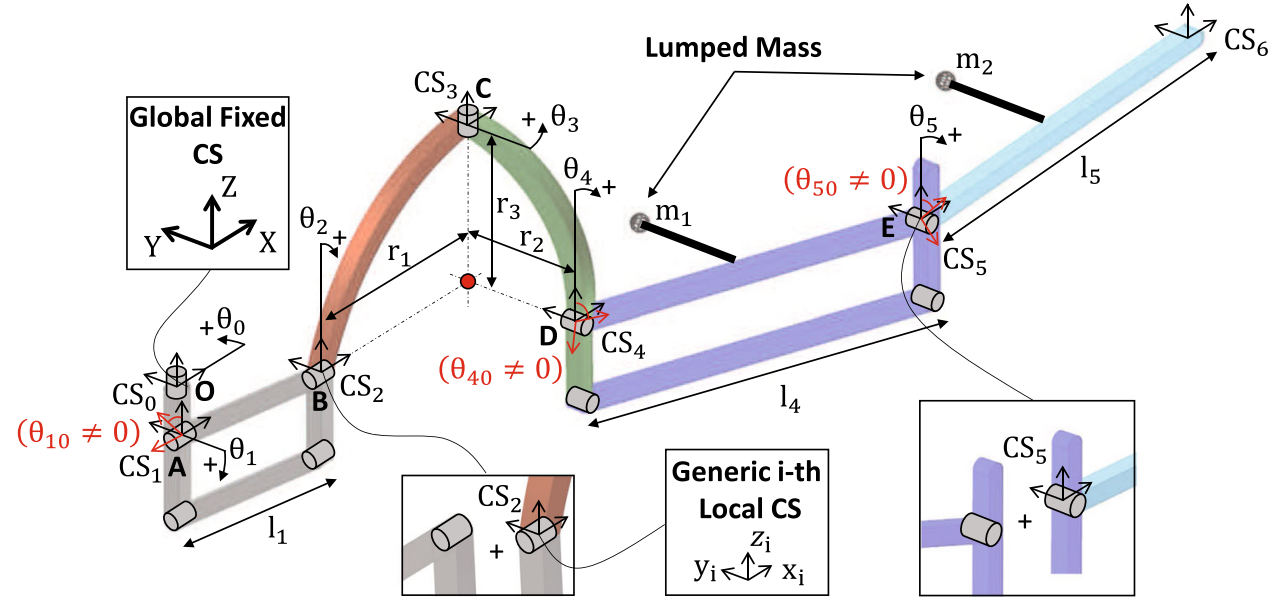

where

$$
\begin{aligned}
& \mathbf{A}_{\mathbf{0}}=\left[\begin{array}{cccc}
\cos \theta_{0} & -\sin \theta_{0} & 0 & 0 \\
\sin \theta_{0} & \cos \theta_{0} & 0 & 0 \\
0 & 0 & 1 & 0 \\
0 & 0 & 0 & 1
\end{array}\right] \\
& \mathbf{A}_{1}=\left[\begin{array}{cccc}
1 & 0 & 0 & 0 \\
0 & 1 & 0 & -l_{1} \cos \theta_{1} \\
0 & 0 & 1 & -l_{1} \sin \theta_{1} \\
0 & 0 & 0 & 1
\end{array}\right] \\
& \mathbf{A}_{2}=\left[\begin{array}{cccc}
1 & 0 & 0 & r_{1} \\
0 & \cos \theta_{2} & -\sin \theta_{2} & -r_{3} \sin \theta_{2} \\
0 & \sin \theta_{2} & \cos \theta_{2} & r_{3} \cos \theta_{2} \\
0 & 0 & 0 & 1
\end{array}\right] \\
& \mathbf{A}_{3}=\left[\begin{array}{cccc}
\cos \theta_{3} & -\sin \theta_{3} & 0 & r_{2} \sin \theta_{3} \\
\sin \theta_{3} & \cos \theta_{3} & 0 & -r_{2} \cos \theta_{3} \\
0 & 0 & 1 & -r_{3} \\
0 & 0 & 0 & 1
\end{array}\right]
\end{aligned}
$$

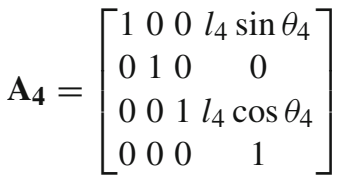

$$
\begin{aligned}
& \mathbf{A}_{5}=\left[\begin{array}{cccc}
\cos \theta_{5} & 0 & \sin \theta_{5} & l_{5} \sin \theta_{5} \\
0 & 1 & 0 & 0 \\
-\sin \theta_{5} & 0 & \cos \theta_{5} & l_{5} \cos \theta_{5} \\
0 & 0 & 0 & 1
\end{array}\right]
\end{aligned}
$$

In accordance with initial mechanism configuration visible in Fig. 8, each rotation angle is defined as $\theta_{i}=\theta_{i, 0}+\Delta \theta_{i}, i \in$ $[0,5]$. The motion parameters of each $\mathrm{R}$ joint are summarized in Table 2, and the resulting exoskeleton design workspace is illustrated in Fig. 9 (where $\theta_{5}=\theta_{5,0}$ for visualization purpose). These operative limits have been chosen with the aim of avoiding kinematic singularities, which otherwise may occur, as clearly pointed out in [43].
Table 1 Geometrical and mass parameters of the virtual model

\begin{tabular}{lll}
\hline Parameter description & Symbol & Value \\
\hline Link1: length & $l_{1}$ & $121 \mathrm{~mm}$ \\
Link2: dimension & $r_{1}$ & $125 \mathrm{~mm}$ \\
Link3: dimension & $r_{2}$ & $75 \mathrm{~mm}$ \\
Link2-3: dimension & $r_{3}$ & $95 \mathrm{~mm}$ \\
Link4: length & $l_{4}$ & $307 \mathrm{~mm}$ \\
Link5: length & $l_{5}$ & $225 \mathrm{~mm}$ \\
Arm: mass & $m_{1}$ & $2.1 \mathrm{~kg}$ \\
Forearm: mass & $m_{2}$ & $1.2 \mathrm{~kg}$ \\
Arm: center of mass position & $a_{4}$ & $0.44 l_{4} \mathrm{~mm}$ \\
Forearm: center of mass position & $a_{5}$ & $0.425 l_{5} \mathrm{~mm}$ \\
\hline
\end{tabular}

Table 2 Motion parameters of the exoskeleton model considered in the analysis

\begin{tabular}{lll}
\hline Joint & $\begin{array}{l}\text { Initial position } \\
\left(\theta_{0}\right)\end{array}$ & $\begin{array}{l}\text { Pos/neg permitted increments } \\
(\Delta \theta)\end{array}$ \\
\hline$R_{0}$ & $0^{\circ}$ & $-15^{\circ}, 20^{\circ}$ \\
$R_{1}$ & $-38^{\circ}$ & $-40^{\circ}, 10^{\circ}$ \\
$R_{2}$ & $0^{\circ}$ & $-60^{\circ}, 60^{\circ}$ \\
$R_{3}$ & $0^{\circ}$ & $-70^{\circ}, 80^{\circ}$ \\
$R_{4}$ & $106^{\circ}$ & $-75^{\circ}, 75^{\circ}$ \\
$R_{5}$ & $90^{\circ}$ & $-140^{\circ}, 0^{\circ}$ \\
\hline
\end{tabular}

\subsection{Static model}

The main purpose of the static analysis is to return, for any generic imposed trajectory of the exoskeleton's end-effector, the torques that need to be externally provided at the six $\mathrm{R}$ joints $\left(R_{i}, \quad i \in[0,5]\right.$ in Fig. 4d) so as to achieve a complete gravity balancing. In the subsequent preliminary model, the masses $m_{1}$ and $m_{2}$ are attached to the Link 4 and Link 5 respectively (with distance $r_{2}$, as visible in Fig. 8). Since $R_{0}$ rotates around the $G C S$ z-axis, the weight will be 


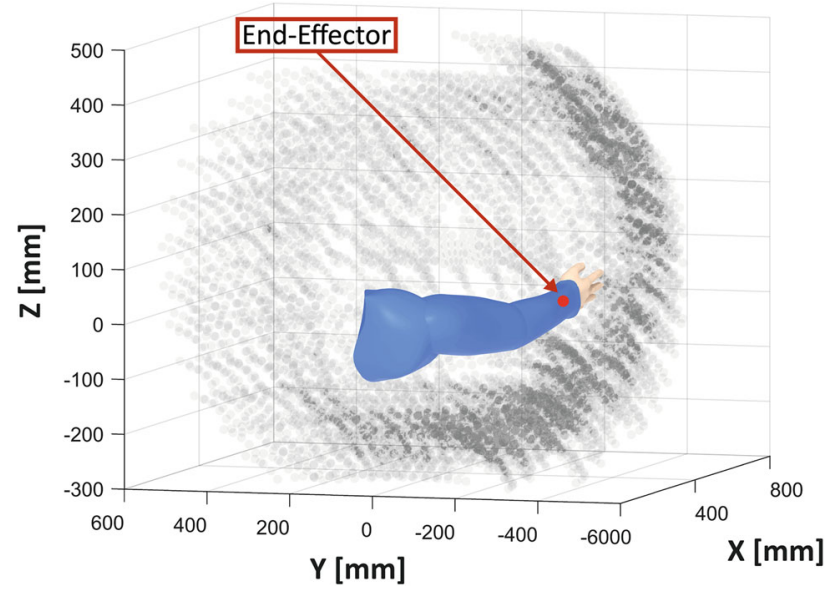

Fig. 9 3D map of the exoskeleton range of motion

equilibrated by the reaction force for any in-operation configuration, removing the need of external balancing actions. As for $R_{1}$, the scapular decoupling mechanism (gray parallelogram in Fig. 8) simplifies the calculation of the gravity torque (as explained in Sect. 3.2), which becomes:

$M_{1}=l_{1}\left(m_{1}+m_{2}\right) g \cos \theta_{1}$

The torque at $R_{2}$ can be formulated as:

$M_{2}=h_{2, m_{1}} m_{1} g+h_{2, m_{2}} m_{2} g$

where, according to Fig. 10a:

$h_{2, m_{1}}=r_{m_{1}} \cos \left(\theta_{2}+\arctan \left(\frac{-v_{4, m_{1}}}{h_{3, m_{1}}}\right)\right)$

$h_{2, m_{2}}=r_{m_{2}} \cos \left(\theta_{2}+\arctan \left(-\frac{v_{4}+v_{5, m_{2}}}{h_{3, m_{2}}}\right)\right)$

$r_{m_{1}}=\sqrt{h_{3, m_{1}}^{2}+v_{4, m_{1}}^{2}}$

$r_{m_{2}}=\sqrt{h_{3, m_{2}}^{2}+\left(v_{4}+v_{5, m_{2}}\right)^{2}}$

and according to Fig. 10b and c:

$$
\begin{aligned}
& h_{3, m_{1}}=h_{4, m_{1}} \sin \theta_{3} \\
& h_{3, m_{2}}=\left(h_{4}+h_{5, m_{2}}\right) \sin \theta_{3} \\
& h_{4, m_{1}}=a_{4} \sin \theta_{4} \\
& h_{4}=l_{4} \sin \theta_{4} \\
& h_{5, m_{2}}=a_{5} \sin \theta_{5} \\
& v_{4, m_{1}}=-a_{4} \cos \theta_{4} \\
& v_{4}=-l_{4} \cos \theta_{4} \\
& v_{5, m_{2}}=-a_{5} \cos \theta_{5}
\end{aligned}
$$

(a)

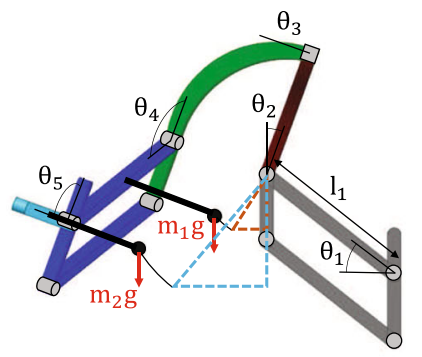

\section{Detailed Schematic for $\mathrm{m} 2$ :}

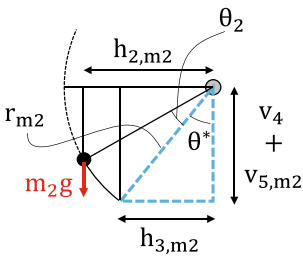

(b)

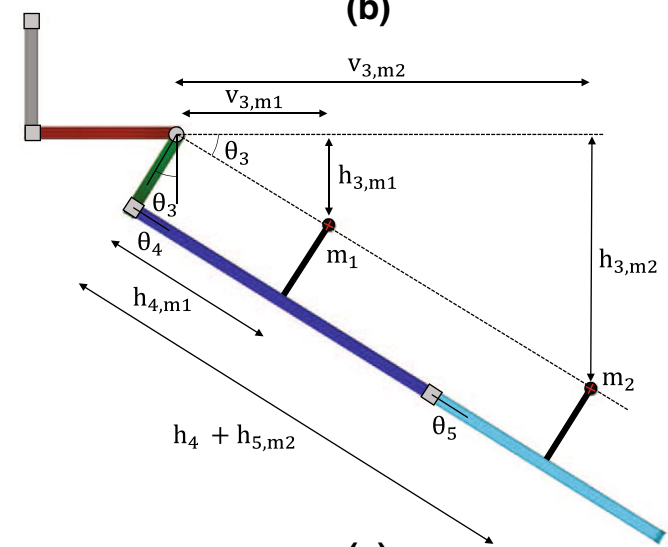

(c)

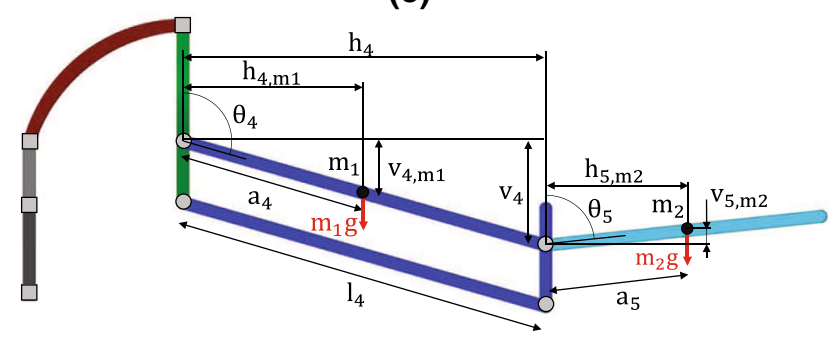

Fig. 10 Mechanism schematics showing the influence of $\theta_{2}$ (a), $\theta_{3}$ (b) and $\theta_{4}-\theta_{5}(\mathbf{c})$ on $M_{2}$

For the remaining $\mathrm{R}$ joints, the analysis can be further simplified by employing the rotation matrices, namely:

$\mathbf{R}_{\mathbf{2}}=\left[\begin{array}{ccc}1 & 0 & 0 \\ 0 \cos \theta_{2} & -\sin \theta_{2} \\ 0 \sin \theta_{2} & \cos \theta_{2}\end{array}\right]$
$\mathbf{R}_{\mathbf{3}}=\left[\begin{array}{ccc}\cos \theta_{3}-\sin \theta_{3} & 0 \\ \sin \theta_{3} & \cos \theta_{3} & 0 \\ 0 & 0 & 1\end{array}\right]$
$\mathbf{R}_{\mathbf{4}}=\left[\begin{array}{ccc}\cos \theta_{4} & 0 & \sin \theta_{4} \\ 0 & 1 & 0 \\ -\sin \theta_{4} & 0 & \cos \theta_{4}\end{array}\right]$
$\mathbf{R}_{5}=\left[\begin{array}{ccc}\cos \theta_{5} & 0 & \sin \theta_{5} \\ 0 & 1 & 0 \\ -\sin \theta_{5} & 0 & \cos \theta_{5}\end{array}\right]$ 
In fact, by defining the forces in the $G C S$ via the vectors $\mathbf{f}_{\mathbf{1}}=\left[\begin{array}{lll}0 & 0 & -m_{1} g\end{array}\right]^{T}$ and $\mathbf{f}_{\mathbf{2}}=\left[\begin{array}{lll}0 & 0 & -m_{2} g\end{array}\right]^{T}$, and by applying the above rotations, the resulting force vectors in the local coordinate systems become:

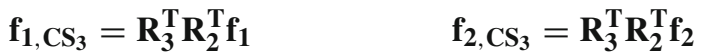

$$
\begin{aligned}
& \mathbf{f}_{1, \mathbf{C S}_{4}}=\mathbf{R}_{\mathbf{4}}^{\mathrm{T}} \mathbf{R}_{\mathbf{3}}^{\mathrm{T}} \mathbf{R}_{\mathbf{2}}^{\mathrm{T}} \mathbf{f}_{\mathbf{1}} \quad \mathbf{f}_{\mathbf{2}, \mathrm{CS}_{4}}=\mathbf{R}_{\mathbf{4}}^{\mathrm{T}} \mathbf{R}_{\mathbf{3}}^{\mathrm{T}} \mathbf{R}_{\mathbf{2}}^{\mathrm{T}} \mathbf{f}_{\mathbf{2}}
\end{aligned}
$$

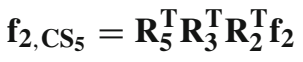

Then, being each of these vectors in the form $\mathbf{f}_{\mathbf{m}, \mathbf{C S}_{\mathbf{n}}}=$ $\left[f_{m x, C S_{n}} f_{m y, C S_{n}} f_{m z, C S_{n}}\right]^{T}$, with $m \in[1,2]$ and $n \in[3,5]$, the torques at joints $R_{3}, R_{4}$ and $R_{5}$ can be found as follows:

$$
\begin{aligned}
& M_{3}=f_{1 y, C S_{3}} \sqrt{v_{3, m_{1}}{ }^{2}+h_{3, m_{1}}{ }^{2}} \\
& +f_{2 y, C S_{3}} \sqrt{v_{3, m_{2}}{ }^{2}+h_{3, m_{2}}{ }^{2}} \\
& M_{4}=f_{1 x, C S_{4}} a_{4}+f_{2 x, C S_{4} l_{4}} \\
& M_{5}=f_{2 x, C S_{5}} a_{5}
\end{aligned}
$$

where

$v_{3, m_{1}}=h_{4, m_{1}} \cos \theta_{3}$

$v_{3, m_{2}}=\left(h_{4}+h_{5, m_{2}}\right) \cos \theta_{3}$

Once the expressions of $M_{i}, i \in[1,5]$ are available, one may choose to design an active balancing system consisting of five actuators. However, from a rapid overview of the proposed static model, it can be noted that the decoupling mechanisms have introduced two important simplifications, namely:

- A contribute $M_{1}$ which is function of the only angle $\theta_{1}$ (see Eq. 20) and can be balanced via a simple passive element;

- A straightforward procedure for the synthesis of the passive springs (see Sect. 3), to be placed at joints $R_{1}, R_{4}$ and $R_{5}$.

Building upon these considerations, in this paper three NZFL springs are included in the mechanical design of the exoskeleton. Their stiffness constants are defined as follows:

$$
\begin{aligned}
& k_{1}=\frac{l_{1}\left(m_{1}+m_{2}\right) g}{b_{1} c_{1}\left(1-\frac{d_{01}}{\sqrt{b_{1}^{2}+c_{1}^{2}}}\right)} \\
& k_{4}=\frac{a_{4} m_{1} g+l_{4} m_{2} g}{b_{4} c_{4}\left(1-\frac{d_{04}}{\sqrt{b_{4}^{2}+c_{4}^{2}}}\right)} \\
& k_{5}=\frac{a_{5} m_{2} g}{b_{5} c_{5}\left(1-\frac{d_{05}}{\sqrt{b_{5}^{2}+c_{5}^{2}}}\right)}
\end{aligned}
$$

where, $b_{1}, b_{4}, b_{5}, c_{1}, c_{4}, c_{5}$ are the installation distances, as visible in Fig. 7 , whereas $d_{01}, d_{04}, d_{05}$ are the initial free lengths of the springs. A part from $M_{1}$, which falls into the planar case study analyzed in Sect. 3.2, in the specific case of $M_{4}$ and $M_{5}$ a complete balancing cannot be achieved via the only use of passive springs due to the spatial motions of the second decoupling mechanism (arm and forearm) and, therefore, the motors are still necessary. The torques provided by the springs are:

$M_{k_{1}}=\frac{k_{1} b_{1} c_{1} \cos \theta_{1}\left(d_{1}-d_{01}\right)}{d_{1}}$

$M_{k_{4}}=\frac{k_{4} b_{4} c_{4} \cos \theta_{4}\left(d_{4}-d_{04}\right)}{d_{4}}$

$M_{k_{5}}=\frac{k_{5} b_{5} c_{5} \cos \theta_{5}\left(d_{5}-d_{05}\right)}{d_{5}}$

where, $d_{1}, d_{4}, d_{5}$ are to be evaluated with the cosine theorem. Therefore, the resulting torques after spring balancing are:

$\begin{aligned} M_{1, b} & =M_{1}-M_{k_{1}} \\ M_{4, b} & =M_{4}-M_{k_{4}} \\ M_{5, b} & =M_{5}-M_{k_{5}}\end{aligned}$

\subsection{Numerical validation}

To validate the previous analysis, a 3D solid model of the exoskeleton has been tested in RecurDyn environment by imposing three overhead movements, shown in Fig. 11, within the considered workspace. The model is analyzed by enforcing a set of rotations $\left(\Delta \theta_{i}, i \in[0,5]\right.$, listed in Table 3 ) and by measuring the resulting reaction torques at each $\mathrm{R}$ joint $\left(R_{i}, i \in[0,5]\right)$. The effect of both ZFL and NZFL springs is modeled through the RecurDyn spring ele-

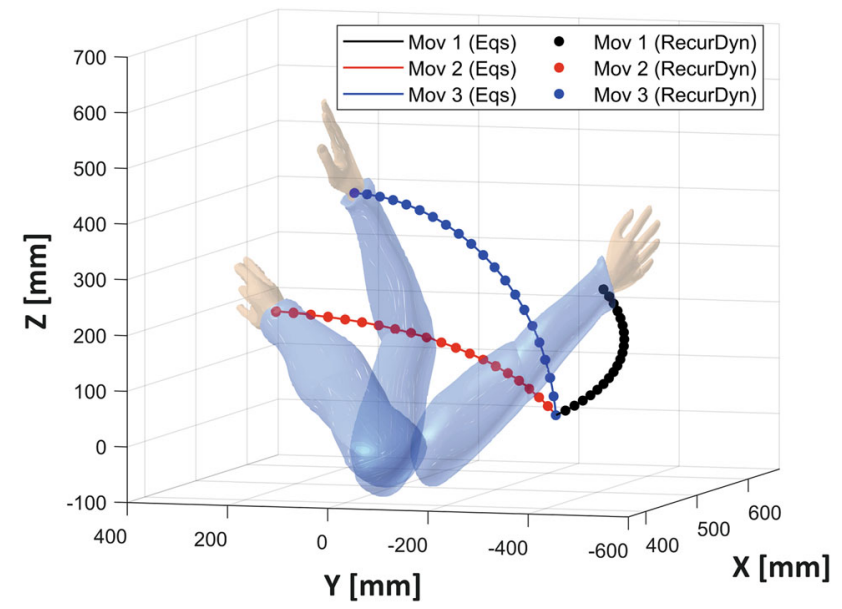

Fig. 11 3D animations of the tested operator's movements 
Table 3 Rotations applied in the simulations

\begin{tabular}{lllllll}
\hline & $\Delta \theta_{0}$ & $\Delta \theta_{1}$ & $\Delta \theta_{2}$ & $\Delta \theta_{3}$ & $\Delta \theta_{4}$ & $\boldsymbol{\Delta} \boldsymbol{\theta}_{\mathbf{5}}$ \\
\hline Mov 1 & $-5^{\circ}$ & $-10^{\circ}$ & $-20^{\circ}$ & $-40^{\circ}$ & $-20^{\circ}$ & $-10^{\circ}$ \\
Mov 2 & $10^{\circ}$ & $-10^{\circ}$ & $-15^{\circ}$ & $35^{\circ}$ & $-30^{\circ}$ & $-25^{\circ}$ \\
Mov 3 & $0^{\circ}$ & $-15^{\circ}$ & $-5^{\circ}$ & $15^{\circ}$ & $-45^{\circ}$ & $-100^{\circ}$ \\
\hline
\end{tabular}

ments. The considered parameters are $b_{1}=0.5 l_{1}, b_{4}=a_{4}$, $b_{5}=a_{5}, c_{1}=c_{4}=c_{5}=30 \mathrm{~mm}, d_{01}=20 \mathrm{~mm}, d_{04}=50$ $\mathrm{mm}$ and $d_{05}=35 \mathrm{~mm}$, with $l_{1}, a_{4}$ and $a_{5}$ as in Table 1 . To exclude the inertial loads, a total simulation time of $5 \mathrm{~s}$ is imposed in the MBD simulations. The results of the three analysis are reported in Figs. 12, 13, 14, 15 and 16. Overall, the models show good agreement, being the medium error between them lower than $0.2 \%$. The main outcomes of this test are as follows:

- The passive elements have a remarkable influence on the computed behaviors, as it may be noticed by comparing $M_{1}-M_{1, b}, M_{4}-M_{4, b}$ and $M_{5}-M_{5, b}$ in Figs. 12,15 and 16. For the totality of the cases, the springs provided large reductions of the reaction torques at joints $R_{1}, R_{4}$ and $R_{5}$.

- As expected, the ZFL springs ensure better performance than the NZFL, i.e. almost null trends in many areas of the plots. Even so, the parameters of the NZFL springs may be further refined through an optimization process to possibly increase the balancing accuracy. The presence of an efficient analytical model surely promotes this approach.

As for the motor selection, by taking into account the contributes of the NZFL springs, the maximum reached torque levels are $650 \mathrm{Nmm}, 4500 \mathrm{Nmm}, 1960 \mathrm{Nmm}, 435 \mathrm{Nmm}$ and $135 \mathrm{Nmm}$ for $M_{1, b}, M_{2}, M_{3}, M_{4, b}$ and $M_{5, b}$ respectively. These values fall within the operative range of the FLA-series by Harmonic Drive SE. In particular, the FLA-11A-50FB and FLA-14A-100FB can satisfy the requirements at $R_{4}-R_{5}$ and at $R_{2}-R_{3}$ respectively.

The reported virtual prototypes represent an effective interactive design tool for the evaluation of the design variants since early design stages and, if properly integrated within modern simulation environments, they allow to quickly identify the best exoskeleton configuration. For instance, by coding the reported analytical model in Matlab, a single candidate can be solved in less than $1 \mathrm{~s}$, promoting the execution of parametric studies involving a large number of candidates. In this way, the final exoskeleton design can be reached, based on the specified motion and compensation requirements, through optimization approaches.

(a)

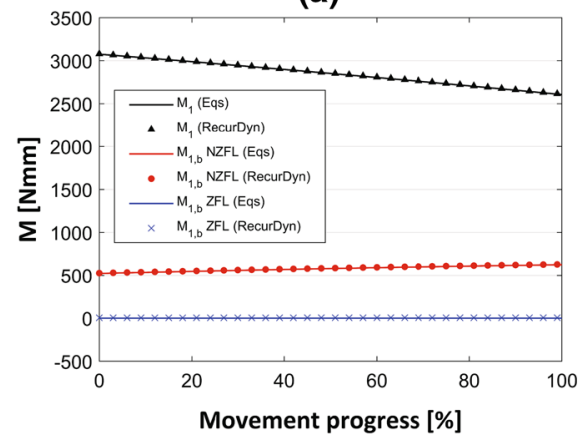

(b)

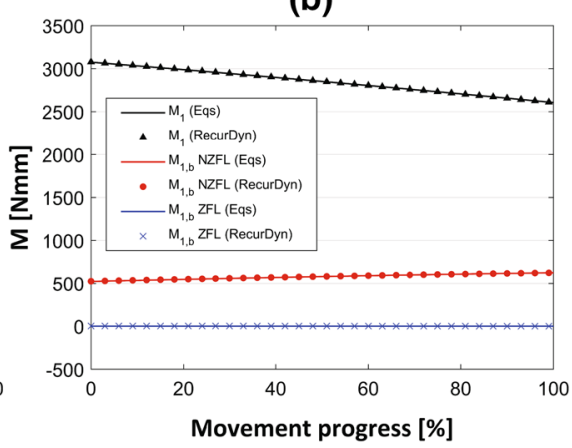

(c)

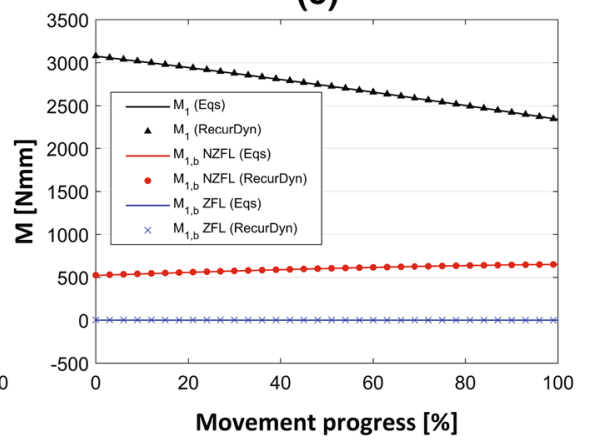

Fig. 12 Registered reaction torques at $R_{1}$ during the first (a), second (b) and third (c) imposed movements

(a)

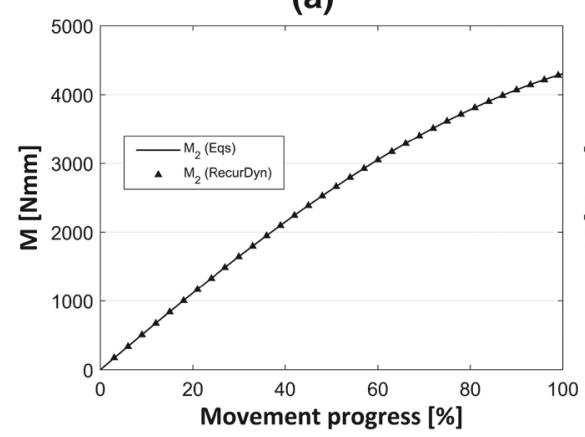

(b)

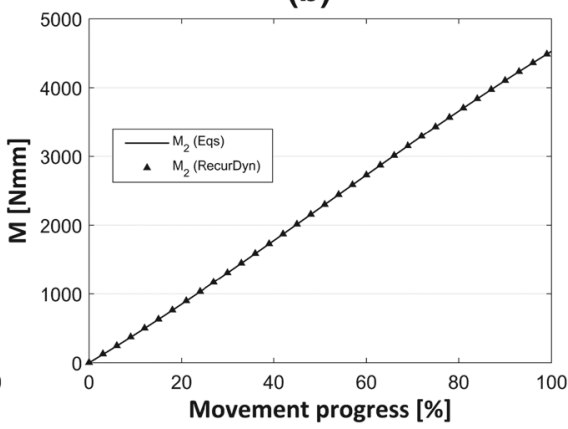

(c)

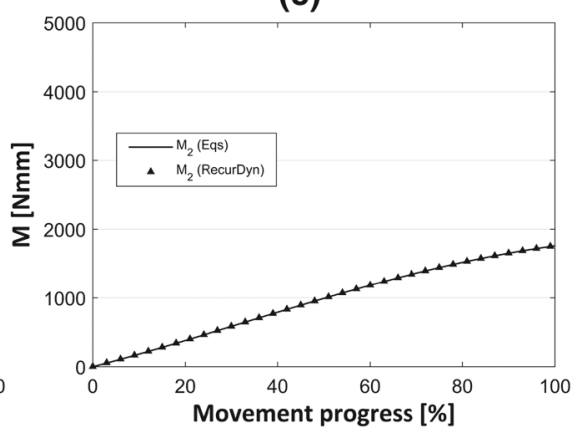

Fig. 13 Registered reaction torque at $R_{2}$ during the first (a), second (b) and third (c) imposed movements 
(a)

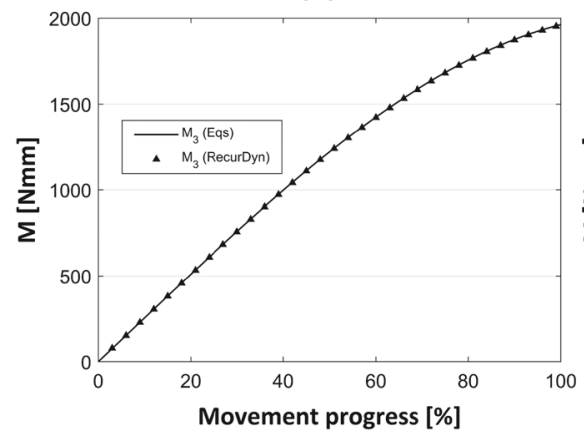

(b)

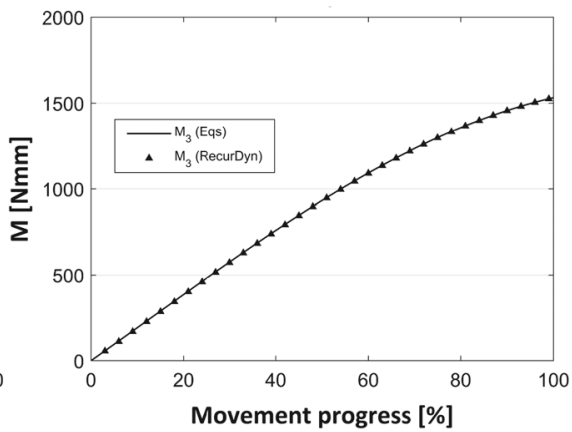

(c)

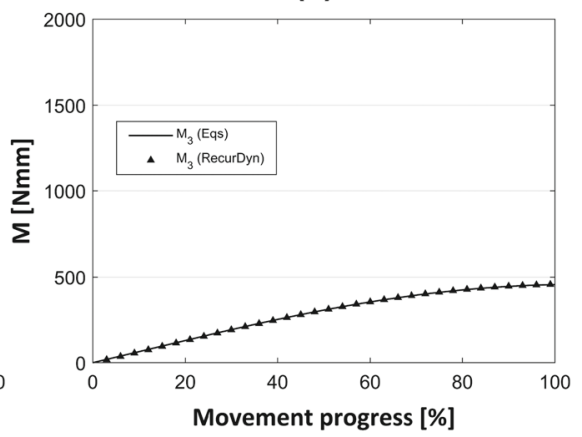

Fig. 14 Registered reaction torque at $R_{3}$ during the first (a), second (b) and third (c) imposed movements

(a)

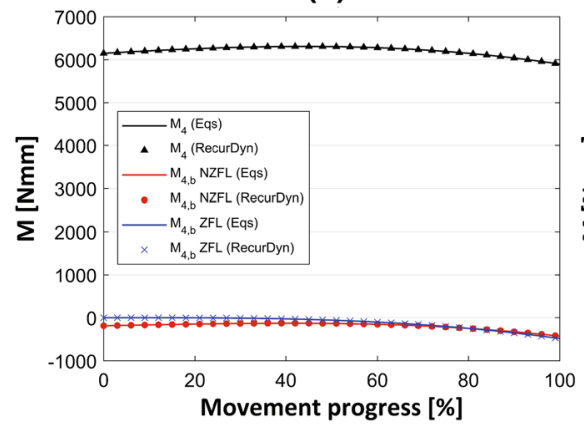

(b)

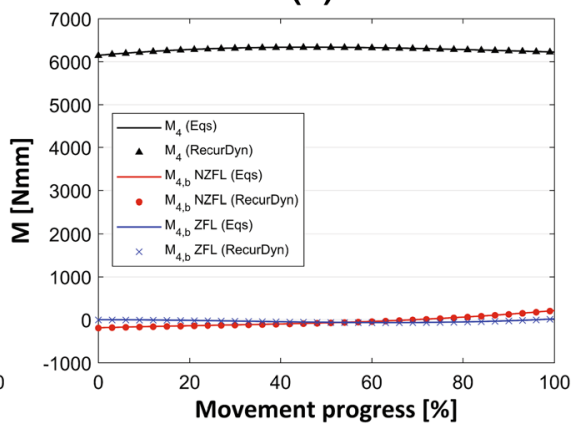

(c)

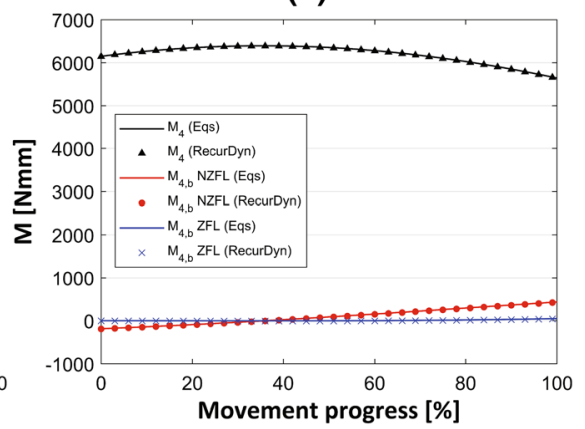

Fig. 15 Registered reaction torques at $R_{4}$ during the first (a), second (b) and third (c) imposed movements

(a)

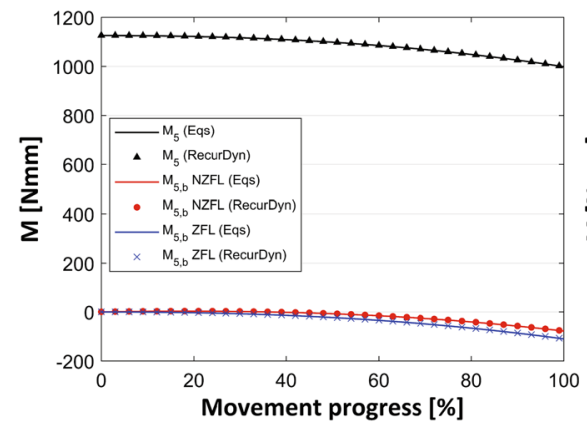

(b)

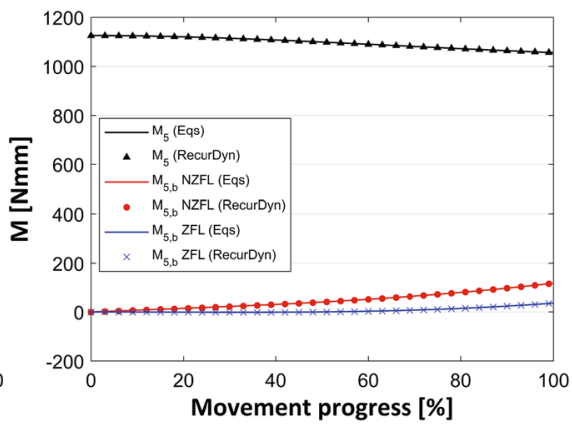

(c)

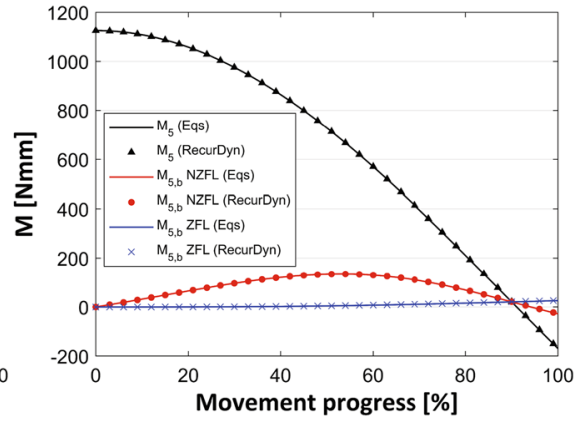

Fig. 16 Registered reaction torques at $R_{5}$ during the first (a), second (b) and third (c) imposed movements

\section{Conclusions}

In this paper, the conceptual design and the virtual prototyping of an upper limb exoskeleton for assisted operations in industrial and healthcare environments is reported. This research has been motivated by the need to provide a simple, low cost, portable device that may be worn to prevent muscle pains during repetitive tasks or to facilitate medical treatments. In the last decades, a number of exoskeletons have been released, generally divided into research proto- types and industrial-oriented prototypes. The former present higher motion capabilities, whereas the latter rely on a compact and wearable structure. The proposed solution has been conceived with the aim of combining these important features. The mechanism consists of a 6 DoFs serial chain with two decoupling parallelograms, and provides the gravity balancing of the human upper limbs through the combined action of four electric motors and three NZFL springs. After a detailed description of the design methods, the paper focused on the kinematic and static analysis of the exoskeleton. An 
analytical model has been developed and then validated via a commercial MBD package. Three different overhead movements were simulated and the achieved results allowed for a proper motor selection. The interactive simulations of the exoskeleton showed that while the use of analytical approaches may be preferable to manage a large number of design parameters, the detailed animations made available by the MBD tool interface allow to visualize the exoskeleton's complex 3D motion and to correct possible errors without screening large amounts of numerical data.

The presented models will be of primary importance for future developments, e.g. for the controller rapid prototyping and for the definition of event-based simulation environments (virtual and augmented reality), which are valuable methods to conduct ergonomic assessments and also to define training sessions for industrial operators and patients.

Funding Open access funding provided by Università degli Studi di Genova within the CRUI-CARE Agreement. This research was funded by University of Genova grant - COSMET, COmpliant Shell-based mechanisms for MEdical Technologies.

Open Access This article is licensed under a Creative Commons Attribution 4.0 International License, which permits use, sharing, adaptation, distribution and reproduction in any medium or format, as long as you give appropriate credit to the original author(s) and the source, provide a link to the Creative Commons licence, and indicate if changes were made. The images or other third party material in this article are included in the article's Creative Commons licence, unless indicated otherwise in a credit line to the material. If material is not included in the article's Creative Commons licence and your intended use is not permitted by statutory regulation or exceeds the permitted use, you will need to obtain permission directly from the copyright holder. To view a copy of this licence, visit http://creativecomm ons.org/licenses/by/4.0/.

\section{References}

1. Gunn, M., Shank, T.M., Eppes, M., Hossain, J., Rahman, T.: User evaluation of a dynamic arm orthosis for people with neuromuscular disorders. IEEE Trans. Neural Syst. Rehabil. Eng. 24(12), 1277-1283 (2015)

2. Hasegawa, Y., Oura, S., Takahashi, J.: Exoskeletal meal assistance system (EMAS II) for patients with progressive muscular disease. Adv. Robot. 27(18), 1385-1398 (2013)

3. Trigili, E., Crea, S., Moisè, M., Baldoni, A., Cempini, M., Ercolini, G., Marconi, D., Posteraro, F., Carrozza, M.C., Vitiello, N.: Design and experimental characterization of a shoulder-elbow exoskeleton with compliant joints for post-stroke rehabilitation. IEEE/ASME Trans. Mechatron. 24(4), 1485-1496 (2019)

4. Wu, Q., Wang, X., Du, F.: Development and analysis of a gravitybalanced exoskeleton for active rehabilitation training of upper limb. Proc. Inst. Mech. Eng. Part C J. Mech. Eng. Sci. 230(20), 3777-3790 (2016)

5. Hyun, D.J., Bae, K., Kim, K., Nam, S., Lee, D.-H.: A light-weight passive upper arm assistive exoskeleton based on multi-linkage spring-energy dissipation mechanism for overhead tasks. Robot. Auton. Syst. 122, 103309 (2019)
6. Ebrahimi, A.: Stuttgart exo-jacket: an exoskeleton for industrial upper body applications. In: International Conference on Human System Interactions (HSI), pp 258-263 (2017)

7. Yu, H., Choi, I.S., Han, K.-L., Choi, J.Y., Chung, G., Suh, J.: Development of a upper-limb exoskeleton robot for refractory construction. Control. Eng. Pract. 72, 104-113 (2018)

8. Bosch, T., van Eck, J., Knitel, K., de Looze, M.: The effects of a passive exoskeleton on muscle activity, discomfort and endurance time in forward bending work. Appl. Ergon. 54, 212-217 (2016)

9. Rahman, T., Sample, W., Seliktar, R., Scavina, M.T., Clark, A.L., Moran, K., Alexander, M.A.: Design and testing of a functional arm orthosis in patients with neuromuscular diseases. IEEE Trans. Neural Syst. Rehabil. Eng. 15(2), 244-251 (2007)

10. Javier, A., Bustamante-Bello, M.R., Ramirez-Mendoza, R.A., Navarro-Tuch, S.A., Izquierdo-Reyes, J., Pablos-Hach, J.L.: Smart health: the use of a lower limb exoskeleton in patients with sarcopenia. Int. J. Interact. Des. Manuf. (IJIDeM) 14(4), 1475-1489 (2020)

11. Pacifico, I., Scano, A., Guanziroli, E., Moise, M., Morelli, L., Chiavenna, A., Romo, D., Spada, S., Colombina, G., Molteni, F., et al.: An experimental evaluation of the proto-MATE: A novel ergonomic upper-limb exoskeleton to reduce workers' physical strain. IEEE Robot. Autom. Mag. 27(1), 54-65 (2020)

12. Hernandez-de Menendez, M., Morales-Menendez, R., Escobar, C.A., McGovern, M.: Competencies for industry 4.0. Int. J. Interact. Des. Manuf. (IJIDeM) 14(4), 1511-1524 (2020)

13. MacDougall, W.: Industrie 4.0: Smart manufacturing for the future. Germany Trade \& Invest (2014)

14. Cohen, Y., Naseraldin, H., Chaudhuri, A., Pilati, F.: Assembly systems in industry 4.0 era: a road map to understand assembly 4.0. Int. J. Adv. Manuf. Technol. 105(9), 4037-4054 (2019)

15. Bances, E., Schneider, U., Siegert, J., Bauernhansl, T.: Exoskeletons towards industrie 4.0: benefits and challenges of the IoT communication architecture. Proc. Manuf. 42, 49-56 (2020)

16. Vergnano, A., Berselli, G., Pellicciari, M.: Interactive simulationbased-training tools for manufacturing systems operators: an industrial case study. Int. J. Interact. Des. Manuf. (IJIDeM) 11(4), 785-797 (2017)

17. Gómez, M.M.: Prediction of work-related musculoskeletal discomfort in the meat processing industry using statistical models. Int. J. Ind. Ergon. 75, 102876 (2020)

18. Spallek, M., Kuhn, W., Uibel, S., van Mark, A., Quarcoo, D.: Workrelated musculoskeletal disorders in the automotive industry due to repetitive work-implications for rehabilitation. J. Occup. Med. Toxicol. 5(1), 1-6 (2010)

19. Comau-Mate.: https://mate.comau.com. Accessed: 2020-07-25

20. Levitate-Airframe.: https://levitatetech.com/airframe. Accessed: 2020-07-25

21. Suitx-Shoulderx.: https://suitx.com/shoulderx. Accessed: 2020$07-25$

22. Suitx-Legx.: https://suitx.com/legx. Accessed: 2020-07-25

23. Laevo-Laevo v2.: https://laevo-exoskeletons.com/laevo-v2. Accessed: 2020-07-25

24. Liu, J., Cheng, Y., Zhang, S., Lu, Z., Gao, G.: Design and analysis of a rigid-flexible parallel mechanism for a neck brace. Math. Probl. Eng. 2019, 9014653 (2019). https://doi.org/10.1155/2019/ 9014653

25. Rb3d-Hercule.: https://rb3d.com/en/exoskeletons/exo. Accessed: 2020-07-25

26. Iranzo, S., Piedrabuena, A., Iordanov, D., Martinez-Iranzo, U., Belda-Lois, J.-M.: Ergonomics assessment of passive upper-limb exoskeletons in an automotive assembly plant. Appl. Ergon. 87, $103120(2020)$

27. De Looze, M.P., Bosch, T., Krause, F., Stadler, K.S., O'Sullivan, L.W.: Exoskeletons for industrial application and their potential effects on physical work load. Ergonomics 59(5), 671-681 (2016) 
28. Crowell, H.P., Park, J.-H., Haynes, C.A., Neugebauer, J.M., Boynton, A.C.: Design, evaluation, and research challenges relevant to exoskeletons and exosuits: a 26-year perspective from the US army research laboratory. IISE Trans. Occup. Ergon. Hum. Factors 7(34), 199-212 (2019)

29. Samper-Escudero, J.L., Giménez-Fernandez, A., Sánchez-Urán, M.Á., Ferre, M.: A cable-driven exosuit for upper limb flexion based on fibres compliance. IEEE Access 8, 153297-153310 (2020)

30. Berselli, G., Mammano, G.S., Dragoni, E.: Design of a dielectric elastomer cylindrical actuator with quasi-constant available thrust: modeling procedure and experimental validation. J. Mech. Des. 136(12), 125001 (2014)

31. Palli, G., Berselli, G., Melchiorri, C., Vassura, G.: Design of a variable stiffness actuator based on flexures. J. Mech. Robot. 3(3), 034501 (2011)

32. Varghese, R.J., Lo, B.P.L., Yang, G.-Z.: Design and prototyping of a bio-inspired kinematic sensing suit for the shoulder joint: precursor to a multi-dof shoulder exosuit. IEEE Robot. Autom. Lett. 5(2), 540-547 (2020)

33. Yu, Y., Liang, W.: Manipulability inclusive principle for hip joint assistive mechanism design optimization. Int. J. Adv. Manuf. Technol. 70(5-8), 929-945 (2014)

34. Hsieh, H.-C., Chen, D.-F., Chien, L., Lan, C.-C.: Design of a parallel actuated exoskeleton for adaptive and safe robotic shoulder rehabilitation. IEEE/ASME Trans. Mechatron. 22(5), 2034-2045 (2017)

35. Castro, M.N., Rasmussen, J., Bai, S., Andersen, M.S.: The reachable 3-D workspace volume is a measure of payload and bodymass-index: a quasi-static kinetic assessment. Appl. Ergon. 75, $108-119$ (2019)

36. Lucas, D.B.: Biomechanics of the shoulder joint. Arch. Surg. 107(3), 425-432 (1973)

37. Perry, J.C., Rosen, J., Burns, S.: Upper-limb powered exoskeleton design. IEEE/ASME Trans. Mechatron. 12(4), 408-417 (2007)

38. Wang, X., Song, Q., Wang, X., Liu, P.: Kinematics and dynamics analysis of a 3-DOF upper-limb exoskeleton with an internally rotated elbow joint. Appl. Sci. 8(3), 464 (2018)

39. Zimmermann, Y., Forino, A., Riener, R., Hutter, M.: ANYexo: a versatile and dynamic upper-limb rehabilitation robot. IEEE Robot. Autom. Lett. 4(4), 3649-3656 (2019)

40. Naidu, D., Stopforth, R., Bright, G., and Davrajh, S.: A 7 DOF exoskeleton arm: shoulder, elbow, wrist and hand mechanism for assistance to upper limb disabled individuals. In: IEEE Africon'11, pp. 1-6 (2011)

41. Zeiaee, A., Soltani-Zarrin, R., Langari, R., Tafreshi, R.: Kinematic design optimization of an eight degree-of-freedom upper-limb exoskeleton. Robotica 37(12), 2073-2086 (2019)

42. Sui, D., Fan, J., Jin, H., Cai, X., Zhao, J., Zhu, Y.: Design of a wearable upper-limb exoskeleton for activities assistance of daily living. In: IEEE International Conference on Advanced Intelligent Mechatronics (AIM), pp. 845-850 (2017)

43. Lo, H.S., Xie, S.: Optimization and analysis of a redundant $4 \mathrm{r}$ spherical wrist mechanism for a shoulder exoskeleton. Robotica 32(8), 1191 (2014)

44. Lo, H.S., Xie, S.S.: An upper limb exoskeleton with an optimized $4 \mathrm{r}$ spherical wrist mechanism for the shoulder joint. In: IEEE International Conference on Advanced Intelligent Mechatronics (AIM), pp. 269-274 (2014)

45. Christensen, S., Bai, S.: Kinematic analysis and design of a novel shoulder exoskeleton using a double parallelogram linkage. ASME J. Mech. Robot. 10(4), 041008 (2018). https://doi.org/10.1115/1. 4040132

46. Tiseni, L., Xiloyannis, M., Chiaradia, D., Lotti, N., Solazzi, M., Van der Kooij, H., Frisoli, A., Masia, L.: On the edge between soft and rigid: an assistive shoulder exoskeleton with hyper-redundant kinematics. In: IEEE International Conference on Rehabilitation Robotics (ICORR), pp. 618-624 (2019)

47. Castro, M.N., Rasmussen, J., Andersen, M.S., Bai, S.: A compact 3-DOF shoulder mechanism constructed with scissors linkages for exoskeleton applications. Mech. Mach. Theory 132, 264-278 (2019)

48. Gopura, R., Bandara, D., Kiguchi, K., Mann, G.K.: Developments in hardware systems of active upper-limb exoskeleton robots: a review. Robot. Auton. Syst. 75, 203-220 (2016)

49. Gull, M.A., Bai, S., Bak, T.: A review on design of upper limb exoskeletons. Robotics 9(1), 16 (2020)

50. Sanjuan, J., Castillo, A.D., Padilla, M.A., Quintero, M.C., Gutierrez, E., Sampayo, I.P., Hernandez, J.R., Rahman, M.H.: Cable driven exoskeleton for upper-limb rehabilitation: a design review. Robot. Auton. Syst. 126, 103445 (2020)

51. Taylor, C.L.: The biomechanics of control in upper-extremity prostheses. Artif. Limbs 2(3), 4-25 (1955)

52. Vitiello, N., Cempini, M., Crea, S., Giovacchini, F., Cortese, M., Moisè, M., Posteraro, F., Carrozza, M.C.: Functional design of a powered elbow orthosis toward its clinical employment. IEEE/ASME Trans. Mechatron. 21(4), 1880-1891 (2016)

53. Tschiersky, M., Hekman, E.E., Brouwer, D.M., Herder, J.L.: Gravity balancing flexure springs for an assistive elbow orthosis. IEEE Trans. Med. Robot. Bion. 1(3), 177-188 (2019)

54. Ball, S.J., Brown, I.E., Scott, S.H.: MEDARM: a rehabilitation robot with 5dof at the shoulder complex. In: IEEE international conference on Advanced Intelligent Mechatronics (AIM), pp. 1-6 (2007)

55. Cui, X., Chen, W., Jin, X., Agrawal, S.K.: Design of a 7-DOF cabledriven arm exoskeleton (CAREX-7) and a controller for dexterous motion training or assistance. IEEE/ASME Trans. Mechatron. 22(1), 161-172 (2016)

56. Huysamen, K., Power, V., O'Sullivan, L.: Kinematic and kinetic functional requirements for industrial exoskeletons for lifting tasks and overhead lifting. Ergonomics. 63(7), 818-830 (2020). https:// doi.org/10.1080/00140139.2020.1759698

57. Wang, H., Xu, H., Tian, Y., Tang, H.: $\alpha$-variable adaptive model free control of irehave upper-limb exoskeleton. Adv. Eng. Softw. 148, $102872(2020)$

58. Matthew, R.P., Mica, E.J., Meinhold, W., Loeza, J.A., Tomizuka, M., Bajcsy, R.: Introduction and initial exploration of an active/passive exoskeleton framework for portable assistance. In: IEEE International Conference on Intelligent Robots and Systems (IROS), pp. 5351-5356 (2015)

59. Saenz, J., Behrens, R., Schulenburg, E., et al.: Methods for considering safety in design of robotics applications featuring human-robot collaboration. Int. J. Adv. Manuf. Technol. 107, 2313-2331 (2020). https://doi.org/10.1007/s00170-020-05076-5

60. Cheng, Z., Foong, S., Sun, D., Tan, U.-X.: Towards a multi-dof passive balancing mechanism for upper limbs. In: IEEE International Conference on Rehabilitation Robotics (ICORR), pp. 508-513 (2015)

61. Nef, T., Riener, R.: Shoulder actuation mechanisms for arm rehabilitation exoskeletons. In: IEEE International Conference on Biomedical Robotics and Biomechatronics (BioRob), pp. 862-868 (2008)

62. Herder, J.L.: Energy-Free Systems. Theory, Conception and Design of Statically, Vol. 2 (2001)

63. Nathan, R.: A constant force generation mechanism. J. Mech. Transm. Autom. Des. 107(4), 508-512 (1985)

64. Chu, Y., Kuo, C.: A single-degree-of-freedom self-regulated gravity balancer for adjustable payload. ASME. J. Mech. Robotics. 9(2), 021006 (2017). https://doi.org/10.1115/1.4035561

65. Chew, D.X.H., Wood, K.L., Tan, U.: Design of a passive selfregulating gravity compensator for variable payloads. ASME. J. 
Mech. Des. 141(10), 102302 (2019). https://doi.org/10.1115/1. 4043582

66. Wang, J., Kong, X.: A geometric approach to the static balancing of mechanisms constructed using spherical kinematic chain units. Mech. Mach. Theory 140, 305-320 (2019)

67. Agrawal, A., Agrawal, S.K.: Design of gravity balancing leg orthosis using non-zero free length springs. Mech. Mach. Theory 40(6), 693-709 (2005)

68. Howell, L.L.: Compliant Mechanisms. Wiley, Hoboken (2001)

69. Bilancia, P., Berselli, G., Palli, G.: Virtual and physical prototyping of a beam-based variable stiffness actuator for safe human-machine interaction. Robot. Comput.-Integr. Manuf. 65, 101886 (2020)

70. Berselli, G., Guerra, A., Vassura, G., Andrisano, A.O.: An engineering method for comparing selectively compliant joints in robotic structures. IEEE/ASME Trans. Mechatron. 19(6), 1882$1895(2014)$
71. Berselli, G., Piccinini, M., and Vassura, G.: Comparative evaluation of the selective compliance in elastic joints for robotic structures. In: 2011 IEEE International Conference on Robotics and Automation, pp. 4626-4631 (2011)

72. Dempster, W.T., Gaughran, G.R.: Properties of body segments based on size and weight. Am. J. Anat. 120(1), 33-54 (1967)

73. Siciliano, B., Sciavicco, L., Villani, L., Oriolo, G.: Robotics: Modelling, Planning and Control. Springer, Berlin (2010)

Publisher's Note Springer Nature remains neutral with regard to jurisdictional claims in published maps and institutional affiliations. 